\title{
Questionário de Vitimização Virtual: Propriedades Psicométricas e Descrições de Vitimização Virtual
}

\author{
Margareth Regina Gomes Veríssimo de Faria, Daniela Sacramento Zanini ${ }^{1}$ \\ Pontifícia Universidade Católica de Goiás, Goiânia-GO, Brasil \\ Evandro Morais Peixoto \\ Universidade de Pernambuco, Garanhuns-PE, Brasil
}

\section{RESUMO}

Este estudo objetiva investigar as evidências de validade e precisão de um instrumento de avaliação de violência virtual. A amostra de 433 estudantes de três escolas públicas, com idades entre 11 e 20 anos $(M=13,91, D P=1,40)$, sendo 54,5\% do sexo feminino, responderam ao Questionário de Vitimização Virtual (QVV), que inclui diferentes episódios de violência por meio de tecnologias de comunicação, inclusive o cyberbullying. A escala de medida foi de dois pontos $(0=$ não; $1=\operatorname{sim})$, avaliando as ocorrências de vitimização em dois momentos da vida: nos últimos doze meses e ao longo da vida. Os resultados apontam evidências de validade e precisão satisfatórias para ambos os momentos avaliados. A TRI possibilitou maior compreensão quanto ao conteúdo dos itens e sua relação com o posicionamento dos itens em um contínuo de dificuldade. Os dados indicaram altos índices de violência virtual entre os adolescentes.

Palavras-chaves: violência; validade; precisão; cyberbullying; adolescentes.

ABSTRACT - Virtual Victimization Questionnaire: psychometric properties and descriptions of virtual victimization This study aims to investigate validity evidence and precision of a virtual violence evaluation instrument. The sample consisted of 433 students from three public schools, between 11 and 20 years of age $(M=13.91, S D=1.40)$, with $54.5 \%$ female. Participants responded to the Virtual Victimization Questionnaire (VVQ), which includes different episodes of violence through communication technologies, including cyberbullying. A two-point scale of measurement $(0=$ no; $1=$ yes $)$, evaluated victimization occurrences during two timelines: in the last twelve months and throughout life. Results point to satisfactory validity evidence and precision for both timelines evaluated. The TRI allowed greater understanding of the items' contents and their relation with the positioning of the items in a continuum of difficulty. The data indicated high rates of virtual violence among adolescents.

Keywords: violence; validity; precision; cyberbullying; adolescents.

RESUMEN - Cuestionario de Victimización Virtual: propiedades psicométricas y descripciones de victimización virtual Este estudio pretende investigar las evidencias de validez y precisión de un instrumento de evaluación de violencia virtual. La muestra de 433 estudiantes de tres escuelas públicas, con edades entre 11 y 20 años $(M=13,91, D S=1,40)$, siendo 54,5\% del sexo femenino, contestaron al Cuestionario de Victimización Virtual (QVV), que incluye diferentes episodios de violencia mediante tecnologías que se utilizan para la comunicación, incluso el cyberbullying. La escala de medida fue de dos puntos $(0=$ no, $1=$ sí), evaluando las ocurrencias de victimización en dos momentos de la vida: en los últimos doce meses y a lo largo de la vida. Los resultados indican evidencias de validez y precisión satisfactorias para ambos momentos evaluados. La TRI posibilitó mayor comprensión en cuanto al contenido de los ítems y su relación con el posicionamiento de los ítems en un continuo de dificultad. Los datos indicaron altos índices de violencia virtual entre los adolescentes.

Palabras clave: violencia; validez; precisión; cyberbullying; adolescentes.

Relatórios oficiais (Waiselfisz, 2012, 2015) apontam um aumento crescente dos casos de violência no Brasil, sobretudo entre os 35 milhões de adolescentes entre 10 e 19 anos no país. Além disso, as diferentes formas de violência provocam grande impacto no desenvolvimento desses indivíduos (Brasil, 2005). Segundo Krug, Dahlberg, Mercy, Zwi e Lozano (2002), é nessa faixa etária que as ações violentas estão mais presentes no Brasil e na América Latina, seja como perpetradoras ou por meio da vivência de suas consequências. Além dos tipos convencionais de violência, outro específico tem crescido entre os adolescentes e preocupado os estudiosos da área, a violência virtual (Rondina, Moura, \& Carvalho, 2016; Wendt \& Lisboa, 2013; Wolak, Mitchell, \& Finkelhor, 2006).

Estudos sobre vitimização virtual são realizados desde 1999 por Wolak et al. (2006) esses mesmos autores 
avaliaram o que mudou após seis anos e mostraram várias situações de vitimização que podem ocorrer por meio da internet, classificando-as em solicitações, exposição involuntária a material sexual e perseguição. Seus estudos expuseram que durante conversas na internet muitas crianças e adolescentes receberam convites relacionados a sexo ou foram assediados. Em alguns casos, foram solicitados encontros "off-line" (fora do ambiente virtual), por telefone ou pessoalmente. Para esses autores, os riscos para as crianças são: exposição a solicitações sexuais (um em cada sete jovens); exposição indesejada a material sexual (um em cada três jovens); assédio ou outro comportamento ofensivo dirigido a eles (um em 11 jovens).

Para Van Den Eijnden, Vermulst, van Rooij, Scholte e van de Mheen (2014), a vitimização virtual é tratada como vitimização on-line. Eles investigaram a relação entre as vitimizações, reais e on-line, com problemas psicossociais em uma amostra de 813 adolescentes de 11 a 15 anos. Os resultados indicaram que a solidão previu um aumento da vitimização na vida real e na ansiedade social, além de sugerirem que as interações negativas on-line e na vida real impactaram significativamente no bem-estar desses adolescentes (Van Den Eijnden et al., 2014).

$\mathrm{O}$ aumento da violência virtual pode ser explicado em decorrência das melhorias tecnológicas e do fácil acesso à internet, seja por computadores ou celulares. Segundo os dados do Instituto Brasileiro de Geografia e Estatística (Instituto Brasileiro de Geografia e Estatística, 2017), 77,1\% das pessoas entrevistadas com 10 anos ou mais tinham celular para uso pessoal e 78,9\% acessavam a internet pelo aparelho. Além disso, 92,6\% dos domicílios possuíam celular e $67,9 \%$ dos domicílios particulares tinham acesso à internet. Embora o uso da internet pela população em geral, e mais especificamente por crianças e adolescentes, já fosse significativo em 2008, sabe-se que aumentou vertiginosamente na década subsequente. Esse aspecto trouxe à tona a existência de um novo ambiente, com possibilidades de manifestação dos diferentes problemas vivenciados no mundo real, entre eles a violência que, nesse caso, denomina-se violência virtual ou, em alguns casos, quando perpetrada por jovens contra seus colegas, cyberbullying (Belsey, 2005).

Belsey (2005) afirmou que o cyberbullying utiliza-se de tecnologias de informação e comunicação para a ocorrência de um comportamento intencional, repetido e hostil por um indivíduo ou grupo com o objetivo de prejudicar outras pessoas. No entanto, para Slonje, Smith e Frinsé (2012), defini-lo pode não ser tão claro quanto definir o bullying tradicional devido a dificuldades nos critérios de repetição e desequilíbrio de poder. Rondina et al. (2016) reforçam a importância de se observar que ele se difere do bullying tradicional principalmente por ser um ato intencional e nem sempre o perpetrador estar envolvido no compartilhamento das mensagens, já que outras pessoas podem fazer isso e afetar a pessoa ou o grupo por diversas vezes (Patchin \& Hinduja, 2015). De todo modo, o que se conclui é que a violência virtual, inclusive o cyberbullying, tornou-se mais frequente entre os adolescentes nos dias de hoje.

A violência virtual inclui o cyberbullying em toda a sua diversidade e também outros tipos de violência cometidos via internet, como exposição de imagens íntimas, pornografia infantil, violência sexual virtual, convites relacionados a sexo feitos a crianças e adolescentes, dentre tantas outras formas que causem prejuízo ou violem a dignidade das pessoas, principalmente os considerados incapazes legalmente. O cyberbullying tem tido especial interesse por parte dos pesquisadores, pois ocorre entre pares; ainda, vem sendo investigado em seus meios, forma e tipo de comportamento. Os principais meios utilizados são celulares ou internet. As formas variam entre mensagens de texto, mensagens instantâneas e páginas da web e os comportamentos presentes nessas mensagens envolvem ameaças, rejeição ou exclusão (Slonje et al., 2012).

Tavares (2012) acredita que é importante conhecer as formas lesivas do cyberbullying e seus impactos para haver punição legal para essa forma de agressão. No entanto, acredita-se que esse estudo pode contribuir também para identificar os tipos de violência virtual e suas ocorrências, de maneira a que se criem estratégias de intervenção para prevenir e quebrar o ciclo da violência virtual. Segundo Rondina et al. (2016), os problemas psicológicos associados ao cyberbullying são depressão, solidão ou insegurança e suicídio ou ideação suicida. Stewart, Drescher, Maack, Ebesutani e Young (2014) também investigaram o cyberbullying em 736 alunos de $6^{\mathrm{a}}$ a $12^{\mathrm{a}}$ séries, no Mississippi, e encontraram correlações positivas significativas com ansiedade, depressão e solidão.

O estudo de Hinduja e Patchin (2010) com 2000 alunos que cursavam entre a $6^{\mathrm{a}}$ e a $8^{\mathrm{a}}$ séries nas escolas dos Estados Unidos avaliou comportamentos ofensivos de cyberbullying e as vitimizações sofridas em decorrência dele. Além disso, os autores relacionaram essa vitimização com o suicídio. Cerca de $20 \%$ dos entrevistados que foram vítimas de cyberbullying afirmaram ter pensado em suicídio (19,7\% das mulheres e $20,9 \%$ dos homens), enquanto $19 \%$ tentaram suicídio $(17,9 \%$ das mulheres e $20,2 \%$ dos homens). Ainda sobre as descrições de ocorrências de cyberbullying, os autores avaliaram os índices de agressões e ofensas emitidas pelos participantes e também as vitimizações sofridas. As taxas de prevalência para comportamentos de violência variaram de $9,1 \%$ a $23,1 \%$ para ofensas e de $5,7 \%$ a $18,3 \%$ para vitimização por cyberbullying. A forma de agressão mais comumente relatada foi a seguinte: "Postado algo on-line sobre outra pessoa para fazer os outros rirem" (23,1\%), enquanto a forma mais frequente de vitimização foi "[t]er recebido um $e$ -mail perturbador de alguém que você conhece" (18,3\%).

Os estudos sobre medidas de bullying já são bastante expressivos na literatura (ver, por exemplo, Vessey, Strout, DiFazio, \& Walker, 2014), porém, são 
recentes sobre cyberbullying e estão se aperfeiçoando na literatura internacional (ex. Wolak et al., 2002; Wolak et al., 2006; Hinduja \& Patchin, 2008, 2010; Gradinger, Strohmeier, \& Spiel, 2010), enquanto são mais raros na literatura brasileira.

Oliveira, Lourenço e Senra (2015) concluíram, em sua revisão bibliométrica, que a maioria das produções científicas sobre o tema violência virtual giram em torno da diferenciação entre bullying convencional e cyberbullying. Realmente, essa é uma preocupação importante, pois essa diferenciação é uma das dificuldades encontradas para o avanço na construção de um instrumento de medida.

Martins (2006) afirma que o sucesso de um instrumento se dá quando ele é merecedor de créditos para a solução de um problema de pesquisa. Para isso, devem ser observados alguns cuidados na construção de um instrumento relacionados às evidências de validade e à sua precisão, essa também referida como confiabilidade do instrumento. Medidas confiáveis são replicáveis e consistentes, enquanto medidas com evidências válidas são representações adequadas dos fenômenos psicológicos que se pretende mensurar frente a uma população específica. Volk, Veenstra e Espelage (2017) também apontam alguns cuidados nas pesquisas sobre bullying como, por exemplo, a definição escolhida para se trabalhar na pesquisa e a escolha adequada da amostra. Esses cuidados visam garantir a validade e a confiabilidade do estudo.

Nessa direção, estudos internacionais têm empregado procedimentos estatísticos, como análise fatorial confirmatória e alfa de Cronbach para avaliar a estrutura interna e os indicadores de precisão dos instrumentos (Gradinger et al., 2010; Stewart et al., 2014; Patchin \& Hinduja, 2015), além de relacionarem a avaliação do bullying convencional com cyberbullying. Nesses estudos, os autores recorrem a escalas Likert de cinco pontos: nunca (0), uma vez (1), algumas vezes (2), várias vezes (3) e muitas vezes (4). No estudo de Patchin e Hinduja (2015), as escalas apresentaram fortes evidências de validade com base na estrutura interna e na confiabilidade interna, com alfas superiores a 0,89 . Além disso, são empregadas estatísticas descritivas para avaliar as ocorrências de cyberbullying (Wolak et al. 2006; Hinduja \& Patchin, 2010; Gradinger et al., 2010; Patchin \& Hinduja, 2015).

Stewart et al. (2014) também avaliaram os fatores de confiabilidade da Escala de Cyberbullying e concluíram que o melhor modelo era a representação por um único fator. Dessa forma, os resultados indicaram que a estrutura do cyberbullying é uma construção unidimensional com uma ótima consistência interna ( $\alpha=0,94$ de Cronbach).

A produção científica sobre cyberbullying vem crescendo em todo o mundo e, entre 2008 e 2013 os países que mais publicaram sobre o tema foram Espanha e Estados Unidos (Oliveira et al., 2015). Em geral, os pesquisadores criaram seus próprios itens ou se utilizaram de escalas já existentes ou, ainda, desenvolveram escalas baseadas em itens encontrados em outros instrumentos (Oliveira et al., 2015). Essas características dos estudos sobre bullying têm trazido preocupação para alguns pesquisadores, que orientam sobre alguns procedimentos importantes no estudo sobre esse tipo de violência, ressaltando que a falta desses cuidados pode comprometer a validade e a fidedignidade dos instrumentos (Volk et al., 2017).

Os estudos de Buelga, Cava e Musitu (2010) e Smith et al. (2008) são citados pela revisão de Oliveira et al. (2015) como alguns dos poucos que investigam a extensão das agressões virtuais, avaliando agressões via telefone móvel e o alcance da perseguição proporcionado pela internet nos últimos trinta ou sessenta dias. Em geral, os estudos sobre violência virtual utilizam amostras grandes e selecionadas de forma aleatória, porém, tratam-se de estudos exploratórios e descritivos, sem a intenção de realizar uma análise de evidências de validade e precisão dos instrumentos (Menesini, Nocentini, \& Calussi, 2011; Oliveira et al., 2015).

Portanto, percebe-se que estudos sobre violência virtual existem, mas a grande maioria são estudos internacionais com análises estatísticas descritivas e exploratórias. Assim, esta pesquisa torna-se bastante relevante para a literatura brasileira, pois tem como objetivo a construção e a investigação das evidências de validade e precisão de um instrumento de avaliação de violência virtual, incluindo o cyberbullying. Nesse sentido, este artigo tem por objetivos: a) desenvolver e estimar a estrutura interna e precisão do Questionário de Vitimização Virtual (QVV); b) estimar as propriedades dos itens e dos participantes por meio da TRI; c) descrever o fenômeno vitimização virtual entre estudantes de 12 a 18 anos de Goiânia.

\section{Método}

\section{Participantes}

A amostra foi composta por 433 estudantes oriundos de três escolas públicas estaduais, selecionadas aleatoriamente, da cidade de Goiânia. Os estudantes cursavam os $7^{\circ}, 8^{\circ}$ e $9^{\circ}$ anos, tendo idades entre 11 e 20 anos, com média de 13,91 anos $(D P=1,40)$. Destes, $54,5 \%$ eram do sexo feminino e $45,5 \%$, do sexo masculino.

\section{Instrumento}

Questionário de Vitimização Virtual (QVV). Neste estudo, preferiu-se a avaliação da violência virtual, que inclui únicos e diferentes episódios de violência por meios de tecnologia da informação e da comunicação, inclusive o cyberbullying (violência virtual sistemática). A escala de medida foi de dois pontos $(0=$ não; $1=\operatorname{sim})$, avaliando as ocorrências de vitimização em dois momentos da vida do adolescente: nos últimos doze meses e ao longo da vida. Dessa forma, é possível verificar as ocorrências ao longo de um ano anterior e também em anos anteriores (ao longo da vida). 


\section{Procedimentos}

Desenvolvimento do Questionário. Desenvolveuse um questionário de cinco itens sobre vitimização virtual. Para esse desenvolvimento, procedeu-se à leitura de artigos referentes ao tema. Posteriormente, utilizando-se da Técnica de Grupo Nominal (Deslandes, Mendes, Pires, \& Campos, 2010), reuniu-se um grupo de cinco juízes, todos treinados e com leituras prévias sobre o tema, para discutirem formas de sofrimento ocasionadas pela violência virtual. O resultado dessa discussão foi sintetizado pela autora principal deste trabalho em oito questões, reapresentadas aos juízes em nova reunião. Após nova análise dos juízes, a escala resultou em cinco questões, respondidas em formato check list (sim/não). O mesmo participante respondia essas questões a partir de duas instruções diferentes sobre o mesmo evento, uma relativa ao acontecimento no último ano e a outra se o fato ocorreu alguma vez em sua vida:

Prezado(a) participante, neste instrumento cada item terá duas respostas, uma referente ao acontecimento no último ano e a outra se o fato ocorreu alguma vez em sua vida. Assim, após a leitura de cada frase marque um X em Sim ou Não de cada uma das duas colunas.

A Tabela 1 abaixo apresenta as questões do questionário.

\section{Análise de Dados}

Para estimar a estrutura interna, foi realizada uma análise fatorial exploratória (AFE), com método de estimação Unweightes Lesast Squares (ULS) e rotação oblíqua promax, análises que se basearam em matrizes de correlações tetracóricas. Para a estimação do número de fatores extraídos em cada uma das escalas, foram empregados diferentes critérios, comumente usados na literatura: Goutman-Kaiser (fatores com eigenvalue superiores a um), análise paralela (AP), método Hull e critério de interpretabilidade da solução fatorial (Tabachnick \& Fidell, 2012). Vale destacar que as análises foram realizadas separadamente para as diferentes versões do questionário, a saber: QVV-a respondida com referência no último ano e o QVV-b respondido com referência ao longo da vida. Uma vez estimada a estrutura interna das diferentes versões do questionário, avaliou-se a precisão de cada instrumento por meio dos coeficientes alfa de Cronbach. Essas análises foram realizadas por meio do programa estatístico Factor 10.3 (Lorenzo-Seva \& Ferrando, 2006).

Para avaliar os parâmetros dos itens e dos participantes, empregou-se o modelo Rasch com método de estimação Joint maximum likelihood. Esse modelo postula que a resposta de uma pessoa a um item é função do nível de traço latente da pessoa (theta/ $\theta$ ) e da dificuldade do item (b). Estimar esses parâmetros de maneira independente permite verificar a probabilidade de as pessoas responderem a cada item do instrumento. Assim, os índices de ajuste dos itens referem-se ao sumário dos resíduos entre respostas esperadas pelo modelo e as apresentadas pelas pessoas. Foram avaliados, na presente pesquisa, os seguintes parâmetros dos itens: índices de dificuldade, índices de ajuste (infit, outfit) e índices de correlação item-theta, bem como os parâmetros dos participantes: nível intensidade no construto (theta) e índices de ajuste (infit, outfit) (ver Linacre, 2015). Tais análises foram realizadas por meio do software estatístico Winsteps 3.7 (Linacre, 2015).

De posse de evidências que assegurassem as propriedades psicométricas das versões do QVV, recorreu-se a estatísticas descritivas a fim de descrever a ocorrência do fenômeno vitimização virtual entre estudantes do estado de Goiás. Para tanto, utilizou-se a estatística percentil e comparação de média (Test $t$ de Student) em função do sexo dos participantes e, nesse caso, estabeleceu-se nível de significância igual a 0,05. Essas análises foram realizadas por meio do software estatístico Jasp 8.0.

\section{Considerações Éticas}

Após a aprovação do projeto pelo Comitê de Ética em Pesquisa sob o protocolo número CAAE 0153.0.168.000-11 (CONEP em 14/12/2011), foi realizado o sorteio das escolas que participariam no estudo. Uma vez identificadas, entrou-se em contato com elas e explicaram-se os objetivos da pesquisa. Foram feitas visitas para apresentar o projeto e solicitar autorização para a pesquisa e o agendamento do dia de coleta de dados. A aplicação foi coletiva, em sala de aula, após o consentimento do responsável legal e assentimento por parte do adolescente. Foi lido e entregue o Termo de Consentimento Livre e Esclarecido (TCLE), que foi levado para casa para que os pais e/ou responsáveis o preenchessem. Ainda assim, os autores declaram ter se comprometido com todos os procedimentos éticos, segundo as Resoluções 196/96 e 466/2012 e orientações da American Psychological Association e, por isso, asseguram total responsabilidade ética do estudo.

\section{Resultados e Discussão}

As AFEs foram antecedidas pela avaliação da adequação das matrizes de correlação disponíveis a cada versão dos questionários, por meio das estatísticas Kaiser Meyer-Olkin (KMO) e teste de esfericidade de Bartlett, os quais demostraram adequação das matrizes para a realização das análises: $\mathrm{KMO}=0,729, \chi^{2}$ de Bartlett $=(10) 359,0, p<0,001$, para o QVV-a, e $\mathrm{KMO}=0,779$, $\chi^{2}$ de Bartlett $=(10) 380,0$, $p<0,001$, para o QVV-b. Dessa forma, avaliaram-se os critérios de retenção do número de fatores, conforme apresentado na Tabela 1.

Conforme apresentado na Tabela 1, os diferentes critérios de retenção indicam a adequação da solução fatorial unidimensional, semelhante ao estudo de Menesini et al., (2011) com 1.092 adolescentes italianos, onde as análises estatísticas também demonstraram que o cyberbullying foi mais bem explicado por um único fator. 
Tabela 1

Adequação da Solução Fatorial Unidimensional para os Questionários QVV-a e QVV-b

\begin{tabular}{|c|c|c|c|c|c|c|c|c|c|c|}
\hline \multicolumn{6}{|c|}{ QVV-a } & \multicolumn{5}{|c|}{ QVV-b } \\
\hline \multirow{3}{*}{ Itens } & \multirow{3}{*}{ Eigenvalue } & \multirow{2}{*}{\multicolumn{2}{|c|}{$\begin{array}{c}\text { Análise Paralela } \\
\text { \% de Variância } \\
\text { explicada }\end{array}$}} & \multirow{3}{*}{$\begin{array}{l}\text { Cargas } \\
\text { Fatoriais }\end{array}$} & \multirow{3}{*}{ Comum. } & \multirow{3}{*}{ Eigenvalue } & \multirow{2}{*}{\multicolumn{2}{|c|}{$\begin{array}{c}\text { Análise Paralela } \\
\text { \% de Variância } \\
\text { explicada }\end{array}$}} & \multirow{3}{*}{$\begin{array}{l}\text { Cargas } \\
\text { Fatoriais }\end{array}$} & \multirow{3}{*}{ Comum. } \\
\hline & & & & & & & & & & \\
\hline & & $\begin{array}{l}\text { Dados } \\
\text { Reais }\end{array}$ & $\begin{array}{c}\text { Percentil } \\
95 \\
\end{array}$ & & & & $\begin{array}{l}\text { Dados } \\
\text { Reais }\end{array}$ & $\begin{array}{c}\text { Percentil } \\
95 \\
\end{array}$ & & \\
\hline 1 & 3,271 & 71,8 & 51,6 & 0,63 & 0,397 & 3,413 & 76,9 & 52,9 & 0,617 & 0,38 \\
\hline 2 & 0,933 & 17,4 & 35,3 & 0,804 & 0,647 & 0,706 & 13,3 & 36,1 & 0,806 & 0,65 \\
\hline 3 & 0,381 & 6,8 & 25,5 & 0,55 & 0,302 & 0,378 & 8,20 & 24,8 & 0,705 & 0,497 \\
\hline 4 & 0,292 & 4,0 & 17,1 & 0,968 & 0,937 & 0,296 & 1,60 & 17,1 & 0,908 & 0,824 \\
\hline 5 & 0,121 & 0,0 & 0,0 & 0,802 & 0,643 & 0,205 & 0,0 & 0,0 & 0,841 & 0,708 \\
\hline \multicolumn{2}{|c|}{ Alfa de Cronbach } & & & 0,952 & & & & & & 0,914 \\
\hline
\end{tabular}

Nota. Comum.=Comunalidades. Fonte. Própria

Para os dois momentos avaliados neste estudo (QVV-a e QVV-b), apenas o primeiro fator apresentou eigenvalue superior a um (3,271 para o QVV-a) e (3,413 para o QVV-b), bem como porcentagem de variância explicada, obtida por meio de dados reais superiores àqueles obtidos através das matrizes aleatórias (71,8 contra 51,6\% para o QVV-a) e (76,9 contra 52,9\% para o QVV-b). Tais resultados foram ainda confirmados pelo método Hull ao indicar a solução unifatorial como a que apresentou melhor equilíbrio entre índices de ajuste e graus de liberdade (qualidade dos índices de ajuste $=(10) 0,808$, e índices Scree teste $=4,141$, para o QVV-a) e (qualidade dos índices de ajuste $=(10)$ 0,941 e índices Scree teste $=$ 14,120, para o QVV-b).

Ainda na Tabela 1 são apresentadas ambas as soluções unifatoriais, sendo possível observar as cargas fatoriais apresentadas pelos itens comunalidade e índices de consistência interna. Considerando-se que as comunalidades são todas superiores a 0,30, e que as cargas fatoriais são iguais ou superiores a 0,497 , pode-se perceber a adequação do modelo fatorial proposto aos dados disponíveis e a boa qualidade dos itens para avaliar o construto de interesse. Esses resultados confirmam a hipótese de que a variável violência virtual em adolescentes (estudantes), quando medida através das diferentes versões do QVV, é de natureza unidimensional (Stewart et al., 2014).

Por fim, os índices de consistência interna, apresentados na Tabela 1 , superiores a 0,9 indicam que ambas as soluções fatoriais são precisas. Portanto, confere às primeiras evidências de precisão as versões do QVV, haja vista que a literatura especializada considera valores superiores a 0,7 como adequados, iguais ou superiores a 0,8 como bons e iguais ou superiores a 0,9 como desejáveis (Tabachnick \& Fidell, 2012). Em comparação a outros estudos que objetivaram a avaliação dos indicadores de precisão de escalas para avaliar tais características, os resultados observados encontram-se muito próximos ou superiores aos verificados por Stewart et al. (2014).

Uma vez estimadas as primeiras evidências de validade e precisão dos instrumentos, avaliaram-se os parâmetros dos itens e dos participantes por meio do modelo Rasch. Os resultados referentes aos itens são apresentados na Tabela 2, onde se verificam os índices de dificuldade dos itens, os índices de ajustes e a correlação entre item-theta.

Tabela 2

Propriedades dos Itens

\begin{tabular}{|c|c|c|c|c|c|c|c|c|}
\hline \multirow{2}{*}{ Itens } & \multicolumn{4}{|c|}{ QVV-a } & \multicolumn{4}{|c|}{ QVV-b } \\
\hline & $b$ & INFIT & OUTFIT & CORR. I- $\Theta$ & $b$ & INFIT & OUTFIT & CORR. I- $\Theta$ \\
\hline 1 & $-1,93$ & 1,22 & 1,79 & 0,71 & $-1,97$ & 1,22 & 1,83 & 0,71 \\
\hline 2 & 0,22 & 0,9 & 0,84 & 0,69 & 0,34 & 0,95 & 0,9 & 0,70 \\
\hline 3 & 1,25 & 1,26 & 1,58 & 0,49 & 1,32 & 1,13 & 1,24 & 0,60 \\
\hline 4 & $-0,13$ & 0,69 & 0,62 & 0,78 & $-0,28$ & 0,78 & 0,72 & 0,77 \\
\hline 5 & 0,59 & 0,89 & 0,85 & 0,66 & 0,59 & 0,89 & 0,85 & 0,70 \\
\hline Média & 0,0 & 0,99 & 1,14 & & 0,0 & 0,99 & 1,11 & \\
\hline $\mathrm{DP}$ & 1,07 & 0,22 & 0,46 & & 1,11 & 0,16 & 0,4 & \\
\hline Mín. & $-1,93$ & 0,69 & 0,62 & & $-1,97$ & 0,78 & 0,85 & \\
\hline Máx. & 1,25 & 1,22 & 1,79 & & 1,32 & 1,22 & 1,83 & \\
\hline
\end{tabular}

Fonte. Própria 
Por meio das estatísticas descritivas mínimo, máximo e desvio padrão dos índices de dificuldades dos itens, observa-se que, embora com poucos itens, os questionários são capazes de cobrir a avalição de uma importante faixa do traço latente, aproximadamente 3,2 logs para a QVV-a e 3,3 para a QVV-b. Ainda em relação à dificuldade dos itens, verificou-se que o item um - "1- Alguém disse coisas a você por internet ou celular (WhatsApp, sites de relacionamento, mensagem celular) que te magoaram?" - apresentou-se como mais fácil para a amostra estudada, enquanto o item três - "3-Alguém fez alguma brincadeira com você utilizando sua imagem (foto) e a expôs na internet para outras pessoas e isso te deixou chateado?" - foi aquele com menos respostas positivas por ocorrer menos frequentemente. Tais resultados podem ser discutidos à luz do conteúdo dos itens, pois é de se esperar que, para a amostra avalia$\mathrm{da}$, violências como receber de alguém mensagem que o magoem, no âmbito privado, sejam mais corriqueiras quando comparadas à exposição de imagens (fotos) na rede. Além disso, do ponto de vista de quem realiza a violência, há na sociedade mais exploração, pela mídia, de casos em que fotos de terceiros são expostas, fazendo com que essas pessoas tomem mais cuidado, em numa tentativa de não deixar provas de suas ações violentas.

A mesma compreensão pode ser aplicada ao avaliar-se o conteúdo dos itens com níveis de dificuldade intermediária - "4- Alguém te xingou ou falou coisas sobre você na internet que te deixaram triste?", "2- Alguém publicou coisas sobre você na internet [WhatsApp, sites de relacionamento, Twitter, Facebook, etc.] que te fizeram sofrer ou que te deixaram envergonhado?" e "5- Alguém te avaliou mal publicamente via internet que deixou você chateado?" -, apresentados em ordem crescente de dificuldade.

Outra importante questão ao analisar o conteúdo dos itens a partir do contínuo da dificuldade é a percepção dos adolescentes de que níveis mais elevados de violência estariam ligados à exposição de sua imagem por meio da publicação de informações a seu respeito, má avaliação pública e exposição de fotos. Pode-se inferir que tais características são mais evidentes nessa etapa da vida, já que, nessa fase, os adolescentes buscam se inserir e serem aceitos nos grupos.

Em relação aos índices de ajustes dos itens, as estatísticas descritivas apontam para a adequação dos valores de Infit $(M=0,99$, Min. =0,69 e Máx. =1,22, para o QVV-a $)$ e $(M=0,99$, Min=0,78 e Máx=1,22, para o QVV-a), uma vez que se encontram dentro do ponto de corte proposto por Linacre (2015) de 0,5 a 1,5. Isso indica a capacidade de esses itens serem previstos pelo modelo quando os valores de dificuldade do item estão próximos aos níveis de traço latente (theta) das pessoas.
Em relação à estatística outfit, os valores médios indicam adequação dos itens de ambas as versões $(1,14 \mathrm{e}$ 0,99 , respectivamente). Contudo, a estatística máxima demonstra que o item 1 apresenta valores superiores a $1,5 \mathrm{em}$ ambas as versões do instrumento $(1,79$ e 1,83 , respectivamente), o que indica menos probabilidade de previsão da resposta ao item quando há uma grande diferença entre o nível de dificuldade do item e o do nível de theta do sujeito.

Nesse caso, pessoas identificadas pelo modelo com níveis de theta elevado poderiam emitir respostas indicando não terem sofrido a violência virtual apresentada no conteúdo do item ("1- Alguém disse coisas a você por internet ou celular (WhatsApp, sites de relacionamento, mensagem celular) que te magoaram?"). Com base no conteúdo, pode-se inferir que, em razão da violência que envolve a exposição da vítima ser mais valorizada entre os adolescentes, violências que ocorrem no âmbito particular, privado, onde não podem ser observadas por outras pessoas, são mais banalizadas por essa população. Além disso, já é destacado, na literatura especializada, que por ser uma estatística baseada em valores inesperados e periféricos o Outfit é mais sensível à influência de outlier, o que pode justificar a manutenção do item ao modelo de medida (Smith, 1996; Wright \& Linacre, 1994). Por fim, destacam-se os índices elevados de correlação observados entre o escore do item e o nível de theta dos participantes, os quais variavam entre 0,49 e 0,78 , para o QVV-a e entre 0,60 e 0,77 para o QVV-b, o que indica a adequação dos itens em estimar o nível de theta dos sujeitos.

Na Tabela 3 são apresentadas as estatísticas descritivas referentes aos parâmetros das pessoas: níveis de theta e índices de ajustes. Quanto aos itens de theta, os valores médios observados indicam que os participantes apresentam níveis de superiores aos de dificuldade dos itens $(b=1,93$ e thet $a=-0,66$ para o QVV-a) e $(b=1,97$ e theta $=-0,66$ para o QVV-b), demostrando serem itens relativamente fáceis para amostra. Do ponto de vista prático, isso indica que os participantes tendem a endossar as alternativas de respostas correspondentes à experiência da vitimização. Contudo, as estatísticas mínimo e máximo indicam que a amostra foi composta por pessoas com variados níveis theta (entre -1,72 e 1,71 quando avaliados pelo QVV-a, e -1,70 e 1,68 para o QVV-b). Isso indica a capacidade dos instrumentos em estimar diferentes níveis de theta.

Quanto aos índices de ajuste, seus valores médios indicam que, de maneira geral, as respostas dos participantes se ajustaram ao padrão resposta esperado pelo modelo. No entanto, estatísticas mínima e máxima apontam para a existência de pessoas com padrão de resposta inesperado, o que é aceitável, haja vista o número de participantes na amostra. 
Tabela 3

Estatísticas Descritivas dos Parâmetros das Pessoas

\begin{tabular}{|c|c|c|c|c|c|c|}
\hline \multirow{2}{*}{ Itens } & \multicolumn{3}{|c|}{ QVV-a } & \multicolumn{3}{|c|}{ QVV-b } \\
\hline & $\Theta$ & INFIT & OUTFIT & $\Theta$ & INFIT & OUTFIT \\
\hline Média & $-0,66$ & 0,96 & 1,11 & $-0,66$ & 0,96 & 1,14 \\
\hline $\mathrm{DP}$ & 1,18 & 0,6 & 1,36 & 1,15 & 0,62 & 1,36 \\
\hline Mín. & $-1,72$ & 0,4 & 0,26 & $-1,7$ & 0,27 & 0,23 \\
\hline Máx. & 1,71 & 2,44 & 8,2 & 1,68 & 3,16 & 7,67 \\
\hline
\end{tabular}

Fonte. Própria

Por fim, foram avaliados os tipos de violência virtual mais frequentes entre os estudantes, tanto no último ano quanto ao longo da vida. São eles a exposição a conteúdos que magoaram via internet (item 1), seguido de xingamentos ou comentários (item 4). Em seguida, a exposição de conteúdos privados (item 2) e avaliação negativa (item 5). A violência virtual de menor ocorrência foi a exposição de imagens (item 3) (Tabela 4). Tais resultados são semelhantes aos observados por meio da TRI, haja vista que proporcionais aos níveis de dificuldade observados nos itens. A porcentagem de respostas observadas em cada uma das alternativas é apresentada na Tabela 4.

Tabela 4

Descrição dos Índices de Vitimização Virtual para Adolescentes no Último Ano e ao Longo da Vida

\begin{tabular}{|c|c|c|c|c|}
\hline \multirow{2}{*}{ Itens de Vitimização Virtual } & \multicolumn{2}{|c|}{ No último ano } & \multicolumn{2}{|c|}{ Alguma vez em sua vida } \\
\hline & $\% \operatorname{Sim}$ & \% Não & $\% \operatorname{Sim}$ & \% Não \\
\hline Item 1 & 33,6 & 63,4 & 36,3 & 63,7 \\
\hline Item 2 & 15,6 & 84,4 & 17,4 & 82,6 \\
\hline Item 3 & 9,2 & 80,8 & 11,2 & 88,8 \\
\hline Item 4 & 18,5 & 81,5 & 21,8 & 78,2 \\
\hline Item 5 & 13,2 & 86,8 & 15,5 & 84,5 \\
\hline Total & Média & $\mathrm{DP}$ & Min. & Máx \\
\hline No último ano & 0,88 & 1,25 & 0 & 5 \\
\hline Alguma vez em sua vida & 1,04 & 1,45 & 0 & 5 \\
\hline
\end{tabular}

Fonte. Própria

Posteriormente, compararam-se os escores totais apresentados pelos participantes em cada um dos instrumentos por meio do teste t de Student para amostras pareadas. Os participantes apresentam resultados significativamente superiores para o somatório de experiências de vitimização ocorridas ao longo da vida $(M=1,29)$ quando comparadas às experiências vivenciadas no último ano $(M=1,14)(t(452)-6,133, p<0,01)$. Tais resultados estão de acordo com os observados na literatura especializada, pois, conforme indicado por Finkelhor, Ormrod, Turner e Hamby (2005), espera-se que as vitimizações acumuladas ao longo da vida sejam maiores que a quantidade de vitimizações sofridas no último ano. Contudo, alguns tipos de violência são mais frequentes no último ano de vida dos adolescentes. Isso pode ter ocorrido devido às particularidades dessa fase da vida, momento em que os pais deixam de acompanhar seus filhos mais de perto e quando eles já possuem alguma independência, tornando-os mais vulneráveis à violência por pares e à violência sexual (Finkelhor et al., 2005).
Além disso, quando esses adolescentes eram crianças, iniciava-se o avanço da tecnologia da informação e da comunicação, por meio da internet e do telefone móvel, logo, entende-se que eles estão em contato com esses meios de comunicação desde que começaram a ser alfabetizados. Desse modo, pode-se entender que a violência virtual já ocorria em anos anteriores e avança rapidamente devido ao desenvolvimento tecnológico e ao fácil acesso à tecnologia, principalmente aos telefones celulares. No entanto, os estudantes não estão habilitados para lidar com todas as consequências dessa evolução tecnológica, haja vista os crescentes números de violência virtual e os problemas emocionais e psicológicos provocados por essa exposição (White \& Carmody, 2016).

Por fim, comparou-se os escores totais em função dos sexos dos participantes por meio do teste $t$ de Students para amostras independentes. Considerando os resultados em ambas versões do questionário, os participantes não se diferem quanto às variáveis estudadas ( $t$ (392) $1,182, p=0,238$ para o QQVE-a) e $(t(397) 1,317$, $p=0,189$ para o QQVE-a), uma vez que tanto meninos e 
meninas apresentam frequência de índices de violência virtual semelhante.

\section{Considerações Finais}

Os objetivos deste estudo foram desenvolver e estimar a estrutura interna e a precisão do Questionário de Vitimização Virtual (QVV); estimar as propriedades dos itens e dos participantes por meio da TRI; e descrever o fenômeno vitimização virtual entre estudantes do estado de Goiás. Os resultados obtidos mostram as primeiras evidências de validade e precisão de ambas as versões do questionário. Vale destacar que a TRI possibilitou mais compreensão quanto ao conteúdo dos itens e sua relação com o posicionamento deles em um contínuo de dificuldade. Por fim, esta pesquisa possibilitou explorar o fenômeno vitimização virtual entre os participantes da amostra representante da cidade de Goiânia. Dessa maneira, pode-se concluir que os objetivos estabelecidos foram satisfatoriamente alcançados. Por fim, destacam-se algumas das limitações, como a composição de uma amostra restrita a uma cidade da região centro-oeste do país, portanto, sugere-se que outros trabalhos sejam realizados com amostras que representem diferentes regiões brasileiras.

\section{Referências}

Belsey, B. (2005). Cyberbullying: An emerging threat to the "always-on" generation. Recuperado de http://www.cyberbullying.ca

Buelga, S., Cava, M. J., \& Musitu, G. (2010). Cyberbullying: Victimización entre adolescentes a través del teléfono móvil y de internet. Psicothema, 22(4), 784-789. Recuperado de http://www.psicothema.com/PDF/3802.pdf

Brasil (2005). Ministério da Saúde. Secretaria de Vigilância em Saúde. Impacto da violência na saúde dos brasileiros. Brasília: Ministério da Saúde.

Deslandes, S. F., Mendes, C. H. F., Pires, T. de O., \& Campos, D. de S. (2010). Use of the nominal group technique and the delphi method to draw up evaluation indicators for strategies to deal with violence against children and adolescents in Brazil. Revista Brasileira de Saúde Materno Infantil, 10, s29-s37. doi: 10.1590/S1519-38292010000500003

Finkelhor, D., Ormrod, R., Turner, H., \& Hamby S. L. (2005, feb.). The victimization of children and youth: A comprehensive, national survey. Child Maltreatment, 10(1), 5-25. doi: 10.1177/1077559504271287

Gradinger, P., Strohmeier, D., \& Spiel, Ch. (2010). Definition and measurement of cyberbullying. Cyberpsychology: Journal of Psychosocial Research on Cyberspace, 4(2). Recuperado de http://cyberpsychology.eu/view.php?cisloclanku=2010112301\&article $=1$

Hinduja, S., \& Patchin, J. W. (2008). Cyberbullying: An exploratory analysis of factors related to offending and victimization. Deviant Behavior, 29(2), 129-156. doi: 10.1080/01639620701457816.

Hinduja, S., \& Patchin, J. W. (2010). Bullying, cyberbullying, and suicide. Archives of Suicide Research, 14(3), 206-221. doi: 10.1080/13811118.2010.494133

Instituto Brasileiro de Geografia e Estatística (2017). Síntese de indicadores sociais: Uma análise das condições de vida da população brasileira. Recuperado de https://biblioteca.ibge.gov.br/visualizacao/livros/liv101459.pdf

Krug, E. G., Dahlberg, L. L., Mercy, J. A., Zwi, A. B., \& Lozano, R. (2002). Relatório mundial sobre violência e saúde. Genebra: Organização Mundial de Saúde.

Linacre, J. M. (2015). A user's guide to Winsteps Ministep: Rasch-model computer programs. Recuperado de http://www.winsteps.com

Lorenzo-Seva, U., \& Ferrando, P.J. Behavior research methods (2006) 38(1), 88-91. doi: 10.3758/BF03192753

Martins, G. de A. (2006). Sobre confiabilidade e validade. RBGN, 8(20), 1-12. Recuperado de www.spell.org.br/documentos/ver/6471/ sobre-confiabilidade-e-validade

Menesini, E., Nocentini, A., \& Calussi, P. (2011). The measurement of cyberbullying: Dimensional structure and relative item severity and discrimination. Cyberpsychology, behavior, and social networking, 14(5), 267-274. doi: 10.1089/cyber.2010.0002

Oliveira, J. C. C. de, Lourenço, L. M., \& Senra, L. X. (2015). A produção científica sobre o cyberbullying: Uma revisão bibliométrica. Psicologia em Pesquisa, 9(1), 31-39. doi: 10.5327/Z1982-1247201500010005

Patchin, J. W., \& Hinduja, S. (2015). Measuring cyberbullying: Implications for research. Aggression and Violent Behavior, 23, 69-74. doi: 10.1016/j.avb.2015.05.013

Rondina, M. J., Moura, J. L., \& Carvalho, M. D. (2016, jan.-jul.). Cyberbullying: O complexo bullying da era digital. Revista de Saúde Digital e Tecnologias Educacionais, 1(1): 20-41. Recuperado de periodicos.ufc.br/resdite/article/view/4682

Stewart, R. W., Drescher, C. F., Maack, D. J., Ebesutani, C., \& Young, J. (2014). The development and psychometric investigation of the Cyberbullying Scale. Journal of interpersonal violence, 29(12), 2218-2238. doi: 10.1177/0886260513517552

Smith, E. R. (1996). What do connectionism and social psychology offer each other? Journal of Personality and Social Psychology, 70(5), 893-912. doi: 10.1037/0022-3514.70.5.893

Smith, P. K., Mahdavi, J., Carvalho, M., Fisher, S., Russell, S., \& Tippett, N. (2008). Cyberbullying: Its nature and impact in secondary school pupils. Journal of Child Psychology and Psychiatry, 49(4), 376-385. doi: 10.1111/j.1469-7610.2007.01846.x

Slonje, R., Smith, \& P., Frisén, A. (2012). The nature of cyberbullying, and strategies for prevention. Computers in Human Behavior, 29(1), 26-32. doi: 10.1016/j.chb.2012.05.024

Tabachnick, B. G., \& Fidell, L. S. (2012). Using multivariate statistics. Person: New Jersey.

Tavares, H. (2012). Cyberbulling na adolescência. Nascer e Crescer, 21(3), s174-s177. Recuperado de http://www.scielo.mec.pt/scielo. php? script $=$ sci_arttext\&pid $=$ S0872-07542012000300016\&lng $=$ pt\&tlng $=$ en.

Van Den Eijnden, R., Vermulst, A., van Rooij, A. J., Scholte, R., \& van de Mheen, D. (2014). The bidirectional relationships between online victimization and psychosocial problems in adolescents: A comparison with real-life victimization. Journal of Youth and Adolescence, 43(5), 790-802. doi: 10.1007/s10964-013-0003-9 
Vessey, J., Strout, T. D., DiFazio, R. L., \& Walker, A. (2014). Measuring the youth bullying experience: A systematic review of the psychometric properties of available instruments. Journal of School Health, 84(12), 819-843. doi: 10.1111/josh.12210

Volk, A. A., Veenstra, R., \& Espelage, D. L. (2017, sept.). So you want study bullying? Recommendations to enhance the validity, transparency, and compatibility of byllying research. Aggression and Violent Behavior, 36, 34-43. doi: 10.1016/j.avb.2017.07.003

Waiselfisz, J. J. (2012). Mapa da violência 2012: Crianças e adolescentes do Brasil. Ed. 1 ${ }^{\text {a }}$ Edição. Rio de Janeiro: Flacso Brasil.

Waiselfisz, J. J. (2015). Mapa da violência 2015: Adolescentes de 16 e 17 ano no Brasil. $1^{\text {a }}$ Edição. Rio de Janeiro: Flacso Brasil.

Wendt, G. W., \& Lisboa, C. S. M. (2013). Agressão entre pares no espaço virtual: Definições, impactos e desafios do cyberbullying. Psicologia Clínica, 25(1), 73-87. doi: 10.1590/S0103-56652013000100005.

White, W. E., \& Carmody, D. (2016). Preventing online victimization college students' views on intervention and prevention. Journal of interpersonal violence, 33(14), 2291-2307. doi: 1177/0886260515625501

Wolak, J., Mitchell, K.J., and Finkelhor, D. (2002). Close online relationships in a national sample of adolescents: A description. Adolescence, 37(147), 441-456. Recuperado de http://unh.edu/ccrc/pdf/jvq/CV50.pdf

Wolak, J., Mitchell, K., \& Finkelhor, D. (2006). Virtual victimization of youth: Five years later. Of the Crimes Against Children Research Center. New Hampshire: University of New Hampshire.

Wright, B. D., \& Linacre, J. M. (1994). Reasonable mean-square fit values. Rasch Measurement Transactions, 8, 370-371. Recuperado de https:// www.rasch.org/rmt/rmt83b.htm

\section{Sobre os autores}

Margareth Regina Gomes Veríssimo de Faria é psicóloga, doutora em Psicologia pela PUC - Goiás, professora colaboradora do Programa de Pós-Graduação Mestrado e Doutorado em Psicologia da Pontifícia Universidade Católica de Goiás e professora dos cursos de Graduação em Psicologia da PUC Goiás e Unievangélica.

Daniela S. Zanini é psicóloga, doutora em Psicologia Clínica e da Saúde pela Universidad de Barcelona (Espanha), professora do Programa de Pós-graduação Mestrado e Doutorado em Psicologia da Pontifícia Universidade Católica de Goiás e bolsista produtividade CNPq nível 2.

Evandro Morias Peixoto é doutor em Psicologia pela Pontifícia Universidade Católica de Campinas PUCC, docente do Departamento de Psicologia da Universidade de Pernambuco - UPE e coordenador do Laboratório de Avaliação Psicológica e Psicometria - LAPPsi. 